\title{
Dynamic layout algorithms for wireless field hockey strategy system
}

\begin{abstract}
Sensor deployment in the field of sports is providing enormous potential for elevating the playing field standards. This paper presents an enhanced localization algorithm which harnesses the dynamic and reconfiguration attributes of field hockey players on a strategy board; known as the Wireless Field Hockey Strategy System (WiHoc Ver1.0). The proposed algorithm utilized for determining the location of field hockey players overcomes the constraints and confined anchor based WiHoc Ver 1.0. The resolution encompasses the new versatile platform which captures the positional location within the parameters of a hockey pitch and of cooperative nodes to determine the optimal location of anchor positioning to ensure that the achieved accuracy of localization is enhanced. The proposed algorithm was evaluated extensively through discrete - event simulations. The acquired results via the accuracy performance metrics validated the enhanced ability of the proposed algorithm.
\end{abstract}

Keyword: Anchor-based; Anchor-free; Localization; WiHoc ver.1.0 